\title{
All meromorphic solutions of some algebraic differential equations and their applications
}

Wenjun Yuan ${ }^{1,2}$, Yezhou $\mathrm{Li}^{3}$ and Jianming Qii*

\section{"Correspondence:} qijianmingsdju@163.com

${ }^{4}$ Department of Mathematics and Physics, Shanghai Dianji University, Shanghai, 201306, China Full list of author information is available at the end of the article

\begin{abstract}
In this paper, we employ Nevanlinna's value distribution theory to investigate the existence of meromorphic solutions of some algebraic differential equations. We obtain the representations of all meromorphic solutions of certain algebraic differential equations with constant coefficients and dominant term. Many results are the corollaries of our result, and we will give the complex method to find all traveling wave exact solutions of corresponding partial differential equations. As an example, we obtain all meromorphic solutions of the Kuramoto-Sivashinsky equation by using our complex method. Our results show that the complex method provides a powerful mathematical tool for solving great many nonlinear partial differential equations in mathematical physics.

MSC: Primary 30D35; secondary 34A05
\end{abstract}

Keywords: differential equation; exact solution; meromorphic function; elliptic function

\section{Introduction and the main result}

Nonlinear partial differential equations (NLPDEs) are widely used as models to describe many important dynamical systems in various fields of science, particularly in fluid mechanics, solid state physics, plasma physics, and nonlinear optics. Exact solutions of NLPDEs of mathematical physics have attracted significant interest in the literature. Over the last years, much work has been done on the construction of exact solitary wave solutions and periodic wave solutions of nonlinear physical equations. Many methods have been developed by mathematicians and physicists to find special solutions of NLPDEs, such as the inverse scattering method [1], Darboux transformation method [2], Hirota bilinear method [3], Lie group method [4], bifurcation method of dynamic systems [5-7], sine-cosine method [8], tanh-function method [9, 10], Fan-expansion method [11], and homogenous balance method [12]. Practically, there is no unified technique that can be employed to handle all types of nonlinear differential equations. Recently, some authors have found the traveling wave exact solutions of certain NLPDEs by using the complex method.

A meromorphic function $w(z)$ means that $w(z)$ is holomorphic in the complex plane $\mathbb{C}$ except for poles. $\alpha, b, c, c_{i}$, and $c_{i j}$ are constants, which may be different from each other in different place. It is assumed that the reader is familiar with the standard notations and the basic results of Nevanlinna's value distribution theory such as

$$
T(r, f), m(r, f), N(r, f) \bar{N}(r, f), \ldots
$$

(c) 2014 Yuan et al.: licensee Springer. This is an Open Access article distributed under the terms of the Creative Commons Attribution License (http://creativecommons.org/licenses/by/2.0), which permits unrestricted use, distribution, and reproduction in any medium, provided the original work is properly cited. 
For details on Nevanlinna's value distribution theory, please see [13]. We denote by $S(r, f)$ any function satisfying $S(r, f)=o\{T(r, f)\}$, as $r \rightarrow \infty$, possibly outside of a set of finite measure. We say that a meromorphic function $f$ belongs to the class $W$ if $f$ is an elliptic function, or a rational function of $e^{\alpha z}, \alpha \in \mathbb{C}$, or a rational function of $z$.

In order to state our results, we need some concepts and notations.

Set $m \in \mathbb{N}:=\{1,2,3, \ldots\}, r_{j} \in \mathbb{N}_{0}=\mathbb{N} \cup\{0\}, r=\left(r_{0}, r_{1}, \ldots, r_{m}\right), j=0,1, \ldots, m$. We define a differential monomial denoted by

$$
M_{r}[w](z):=[w(z)]^{r_{0}}\left[w^{\prime}(z)\right]^{r_{1}}\left[w^{\prime \prime}(z)\right]^{r_{2}} \cdots\left[w^{(m)}(z)\right]^{r_{m}} .
$$

$p(r):=r_{0}+2 r_{1}+\cdots+(m+1) r_{m}$ and $\operatorname{deg}(M)$ are called the weight and degree of $M_{r}[w]$, respectively.

A differential polynomial $P[w]$ is defined as follows:

$$
P[w]:=\sum_{r \in I} a_{r} M_{r}[w]
$$

where $a_{r}$ are constants and $I$ is a finite index set. The total weight and degree of $P[w]$ are defined by $W(P):=\max _{r \in I}\{p(r)\}$ and $\operatorname{deg}(P):=\max _{r \in I}\left\{\operatorname{deg}\left(M_{r}\right)\right\}$, respectively.

We will consider the following complex ordinary differential equation:

$$
P[w]=w^{n},
$$

where $n \in \mathbb{N}$.

Let $p, q \in \mathbb{N}$. Suppose that equation (1.1) has a meromorphic solution $w$ with at least one pole. We say that equation (1.1) satisfies the $\langle p, q\rangle$ condition if there exactly exist $p$ distinct meromorphic solutions of (1.1) with pole of multiplicity $q$ at $z=0$.

We know that verifying the fact that equation (1.1) satisfies the $\langle p, q\rangle$ condition may be very difficult, and many equations (1.1) do not satisfy the $\langle p, q\rangle$ condition. We say that equation (1.1) satisfies the weak $\langle p, q\rangle$ condition if substituting the Laurent series

$$
w(z)=\sum_{k=-q}^{\infty} c_{k} z^{k}, \quad q>0, c_{-q} \neq 0
$$

into equation (1.1) we can determine $p$ distinct Laurent principle parts as the form

$$
\sum_{k=-q}^{-1} c_{k} z^{k}
$$

In 2006, Eremenko [14] researched the existence of meromorphic solutions of the complex Kuramoto-Sivashinsky equation

$$
v w^{\prime \prime \prime}+b w^{\prime \prime}+\mu w^{\prime}+\frac{w^{2}}{2}+A=0, \quad v \neq 0
$$

where $v, \mu, b$, and $A$ are constants, and obtained that all meromorphic solutions $w$ of equation (1.3) are such that $w \in W$. In 1986, Eremenko [15] investigated the $k$-order Briot- 
Bouquet equations

$$
F\left(w^{(k)}, w\right)=\sum_{i=0}^{n} P_{i}(w)\left(w^{(k)}\right)^{i}=0,
$$

where $P_{i}(w)$ are polynomials with constant coefficients in $w$. He proved that any meromorphic solution $w$ of equation (1.4) belongs to the class $W$ if $w$ has at least one pole and $k$ is odd. In 2009, Eremenko et al. [16] proved the following.

Theorem A If $w$ is a meromorphic solution of equation (1.4) and $w$ has at least one pole, then $w \in W$.

Recently, Yuan et al. [17] obtained the following Theorem B and proposed Conjecture C.

Theorem B Let $p, l, m, n \in \mathbb{N}, \operatorname{deg}(P)<n$, and equation (1.1) satisfy the $\langle p, q\rangle$ condition. Then all meromorphic solutions $w$ of equation (1.1) belong to the class $W$. If for some values of parameters such a solution $w$ exists, then other meromorphic solutions form a one-parametric family $w\left(z-z_{0}\right), z_{0} \in \mathbb{C}$. Furthermore, $w$ with pole at 0 must be one of the following three cases:

(I) Each rational function solution $w:=R(z)$, say $z_{1}=0$, is of the form

$$
R(z)=\sum_{i=1}^{l} \sum_{j=1}^{q} \frac{c_{i j}}{\left(z-z_{i}\right)^{j}}+c_{0},
$$

with $l(\leq p)$ distinct poles of multiplicity $q$.

(II) Each simply periodic solution is a rational function $R(\xi)$ with pole at $\xi_{1}=1$ of $\xi=e^{\alpha z}(\alpha \in \mathbb{C}) . R(\xi)$ has $l(\leq p)$ distinct poles of multiplicity $q$, and is of the form

$$
R(\xi)=\sum_{i=1}^{l} \sum_{j=1}^{q} \frac{c_{i j}}{\left(\xi-\xi_{i}\right)^{j}}+c_{0} .
$$

(III) Each elliptic solution can be written as

$$
\begin{aligned}
w(z)= & \sum_{i=1}^{l-1} \sum_{j=2}^{q} \frac{(-1)^{j} c_{-i j}}{(j-1) !} \frac{d^{j-2}}{d z^{j-2}}\left(\frac{1}{4}\left[\frac{\wp^{\prime}(z)+B_{i}}{\wp(z)-A_{i}}\right]^{2}-\wp(z)\right) \\
& +\sum_{i=1}^{l-1} \frac{c_{-i 1}}{2} \frac{\wp^{\prime}(z)+B_{i}}{\wp(z)-A_{i}}+\sum_{j=2}^{q} \frac{(-1)^{j} c_{-l j}}{(j-1) !} \frac{d^{j-2}}{d z^{j-2}} \wp(z)+c_{0},
\end{aligned}
$$

where $\wp(z)$ is the Weierstrass elliptic function, $c_{-i j}$ are given by $(1.2), B_{i}^{2}=4 A_{i}^{3}-g_{2} A_{i}-$ $g_{3}$ and $\sum_{i=1}^{l} c_{-i 1}=0$. And $w$ has another form which is one of the following three forms.

(1) If $w$ is even and the pole of the Weierstrass elliptic function $\wp(z)$ is the pole of $w$, then $w$ is a rational $Q(\xi)$ of $\xi:=\wp(z)$ and has the form of

$$
Q(\xi)=\sum_{i=1}^{l} \sum_{j=1}^{q} \frac{c_{i j}}{\left(\xi-\xi_{i}\right)^{j}}+\sum_{i=0}^{q_{0}} c_{i} \xi^{i}
$$

where $q_{0} \leq \frac{q}{2}$. If the pole of $\wp(z)$ is not the pole of $w$, then $q_{0}=0$. 
(2) If $w$ is odd, then $\frac{w}{\wp^{\prime}(z)}$ is a rational $Q(\xi)$ of $\xi:=\wp(z)$.

(3) If $w$ is non-odd and non-even, then

$$
w(z)=Q_{1}(\wp(z))+\wp(z)^{\prime} Q_{2}(\wp(z)),
$$

where $Q_{1}(\xi)$ and $Q_{2}(\xi)$ are rational functions with the form of (1.8).

Conjecture C Suppose that $p, m, n \in \mathbb{N}$, equation (1.1) satisfies the weak $\langle p, q\rangle$ condition and $\operatorname{deg}(P)<n$. Then the conclusions of Theorem $\mathrm{B}$ hold.

In this paper, we employ Nevanlinna's value distribution theory to investigate the existence of meromorphic solutions of some algebraic differential equations. We give positive answer to Conjecture $\mathrm{C}$ and obtain the representations of all meromorphic solutions of certain algebraic differential equations with constant coefficients and dominant term. Many results are the corollaries of our result, and we will give the complex method to find all traveling wave exact solutions of corresponding partial differential equations. As an example, we obtain all meromorphic solutions of the Kuramoto-Sivashinsky equation (1.3) by using our complex method. Our results show that the complex method provides a powerful mathematical tool for solving great many nonlinear partial differential equations in mathematical physics.

Our main result is the following Theorem 1.

Theorem 1 Consider an algebraic differential equation

$$
a w^{(m)}+P[w]=w^{n},
$$

where $m, n \in \mathbb{N}, a \neq 0, m$ is an odd integer, and $P[w]$ is a differential polynomial. Suppose that equation (1.9) satisfies the weak $\langle p, q\rangle$ condition, and $p(r)+(q-1) \operatorname{deg}\left(M_{r}\right)<m+q$, $\operatorname{deg}(P)<n$. Then the conclusions of Theorem B hold, i.e., all meromorphic solutions $w$ of equation (1.9) belong to the class $W$. If for some values of parameters such a solution $w$ exists, then other meromorphic solutions form a one-parametric family $w\left(z-z_{0}\right), z_{0} \in \mathbb{C}$. Furthermore, $w$ with pole at 0 must be one of the following three cases:

(I) Each rational function solution $w:=R(z)$, say $z_{1}=0$, is of the form

$$
R(z)=\sum_{i=1}^{l} \sum_{j=1}^{q} \frac{c_{i j}}{\left(z-z_{i}\right)^{j}}+c_{0},
$$

with $l(\leq p)$ distinct poles of multiplicity $q$.

(II) Each simply periodic solution is a rational function $R(\xi)$ with pole at $\xi_{1}=1$ of $\xi=e^{\alpha z}(\alpha \in \mathbb{C}) . R(\xi)$ has $l(\leq p)$ distinct poles of multiplicity $q$, and is of the form

$$
R(\xi)=\sum_{i=1}^{l} \sum_{j=1}^{q} \frac{c_{i j}}{\left(\xi-\xi_{i}\right)^{j}}+c_{0} .
$$


(III) Each elliptic solution can be written as

$$
\begin{aligned}
w(z)= & \sum_{i=1}^{l-1} \sum_{j=2}^{q} \frac{(-1)^{j} c_{-i j}}{(j-1) !} \frac{d^{j-2}}{d z^{j-2}}\left(\frac{1}{4}\left[\frac{\wp^{\prime}(z)+B_{i}}{\wp(z)-A_{i}}\right]^{2}-\wp(z)\right) \\
& +\sum_{i=1}^{l-1} \frac{c_{-i 1}}{2} \frac{\wp^{\prime}(z)+B_{i}}{\wp(z)-A_{i}}+\sum_{j=2}^{q} \frac{(-1)^{j} c_{-l j}}{(j-1) !} \frac{d^{j-2}}{d z^{j-2}} \wp(z)+c_{0},
\end{aligned}
$$

where $\wp(z)$ is the Weierstrass elliptic function, $c_{-i j}$ are given by (1.2),

$B_{i}^{2}=4 A_{i}^{3}-g_{2} A_{i}-g_{3}$, and $\sum_{i=1}^{l} c_{-i 1}=0$. And $w$ has another form which is one of the following three forms.

(1) If $w$ is even and the pole of the Weierstrass elliptic function $\wp(z)$ is the pole of $w$, then $w$ is a rational $Q(\xi)$ of $\xi:=\wp(z)$ and has the form of

$$
Q(\xi)=\sum_{i=1}^{l} \sum_{j=1}^{q} \frac{c_{i j}}{\left(\xi-\xi_{i}\right)^{j}}+\sum_{i=0}^{q_{0}} c_{i} \xi^{i},
$$

where $q_{0} \leq \frac{q}{2}$. If the pole of $\wp(z)$ is not the pole of $w$, then $q_{0}=0$.

(2) If $w$ is odd, then $\frac{w}{\wp^{\prime}(z)}$ is a rational $Q(\xi)$ of $\xi:=\wp(z)$.

(3) If $w$ is non-odd and non-even, then

$$
w(z)=Q_{1}(\wp(z))+\wp(z)^{\prime} Q_{2}(\wp(z)),
$$

where $Q_{1}(\xi)$ and $Q_{2}(\xi)$ are rational functions with the form of (1.8).

Consider the algebraic differential equation

$$
C_{0} w^{\prime \prime \prime}+C_{1} w^{\prime \prime}+C_{2} w w^{\prime}+C_{3} w^{\prime}+C_{4} w^{3}+C_{5} w+C_{6}=C_{7} w^{2}+C_{8} w^{4},
$$

where $C_{0} \neq 0, C_{i}, i=1,2, \ldots, 8$, are constants.

Corollary 2 If $C_{8} \neq 0$ or $C_{8}=0, C_{4} \neq 0$ or $C_{8}=C_{2}=C_{4}=0, C_{7} \neq 0$, then any meromorphic solution $w$ of equation (1.10) belongs to $W$.

Remark 1 In 2010, Conte and Ng [18] obtained the above Corollary 2 by using the different method, in which $C_{8} \neq 0$ and all meromorphic solutions were given. In the later case, equation (1.10) is the same as the Kuramoto-Sivashinsky equation (1.3), and Eremenko [14] pointed out that there are no meromorphic solutions except those found in [19-21].

This paper is organized as follows. In the next section, the preliminary lemmas and the complex method are given. The proof of Theorem 1 will be given in Section 3. All meromorphic solutions of the Kuramoto-Sivashinsky equation (1.3) are derived from our complex method in Section 4. Some conclusions and discussions are given in the final section.

\section{Preliminary lemmas and the complex method}

In order to prove our results, we need the following four lemmas. 
Lemma 1 (Clunie lemma [22, 23]) Let $w$ be a meromorphic solution of an equation $w^{n} P\left(z, w, w^{\prime}, \ldots, w^{(m)}\right)=Q\left(z, w, w^{\prime}, \ldots, w^{(m)}\right)$, where $P$ and $Q$ are two differential polynomials in $w$ and its derivatives $w^{\prime}, \ldots, w^{(m)}$ with meromorphic coefficients $\left\{a_{\lambda} \mid \lambda \in I\right\}$, and $m\left(r, a_{\lambda}\right)=$ $S(r, w)$. If the total degree of $Q\left(z, w, w^{\prime}, \ldots, w^{(m)}\right)$ in $w$ and its derivatives $w^{\prime}, \ldots, w^{(m)}$ satisfy $\operatorname{deg} Q\left(z, w, w^{\prime}, \ldots, w^{(m)}\right) \leq n$, then

$$
m\left(r, P\left(z, w, w^{\prime}, \ldots, w^{(m)}\right)\right)=S(r, w) .
$$

Lemma 2 (Mohon'ko theorem [22, 23]) Let w be a meromorphic function, let

$$
R(z, w):=\frac{P(z, w)}{Q(z, w)}=\frac{\sum_{i=0}^{p} a_{i}(z) w^{i}}{\sum_{j=0}^{q} b_{j}(z) w^{j}}
$$

be an irreducible rational function in $w$ with meromorphic coefficients $a_{i}(z), b_{j}(z)$, and let $T\left(r, a_{i}\right)=S(r, w), T\left(r, b_{j}\right)=S(r, w), i=0, \ldots, p, j=0, \ldots, q$. Then

$$
T(r, R(z, w))=d T(r, w)+S(r, w),
$$

where $d=\max (p, q)$.

Lemma 3 Let $M_{r, m}[w](z):=\prod_{i=0}^{m}\left[w^{(i)}(z)\right]^{r_{i}}$ be a differential monomial with weight and degree $p(r)$ and $\operatorname{deg}(M)$, respectively. Suppose that $w_{1}(z)$ and $w_{2}(z)$ are two functions. Then

$$
M_{r, m}\left[w_{1}\right]-M_{r, m}\left[w_{2}\right]=\sum_{i=0}^{m}\left(w_{1}^{(i)}-w_{2}^{(i)}\right) P_{i, m}\left[w_{1}, w_{2}\right],
$$

where each $P_{i, m}\left[w_{1}, w_{2}\right]=\prod_{k=0}^{i-1}\left[w_{1}^{(k)}\right]^{r_{k}} \prod_{k=i+1}^{m}\left[w_{2}^{(k)}\right]^{r_{k}}\left(\sum_{k=0}^{r_{i}-1}\left[w_{1}^{(i)}\right]^{k}\left[w_{2}^{(i)}\right]^{r_{i}-1-k}\right)(i=0, \ldots, m)$ is a homogenous differential polynomial in $w_{1}$ and $w_{2}$ with the total weight $W\left(P_{i, m}\right)=p(r)-$ $i-1$ and degree $\operatorname{deg}\left(P_{i, m}\right)=\operatorname{deg}(M)-1$, respectively.

Proof When $m=0$, we have

$$
M_{r, 0}\left[w_{1}\right]-M_{r, 0}\left[w_{2}\right]=w_{1}^{r_{0}}-w_{2}^{r_{0}}=\left(w_{1}-w_{2}\right)\left(w_{1}^{r_{0}-1}+w_{1}^{r_{0}-2} w_{2}+\cdots+w_{2}^{r_{0}-1}\right),
$$

which shows that Lemma 3 holds.

Suppose that Lemma 3 holds for all $k \leq m$. Noting

$$
\begin{aligned}
M_{r, m}\left[w_{1}\right]-M_{r, m}\left[w_{2}\right]= & \prod_{i=0}^{m}\left[w_{1}^{(i)}\right]^{r_{i}}-\prod_{i=0}^{m}\left[w_{2}^{(i)}\right]^{r_{i}} \\
= & \prod_{i=0}^{m-1}\left[w_{1}^{(i)}\right]^{r_{i}}\left(\left[w_{1}^{(m)}\right]^{r_{m}}-\left[w_{2}^{(m)}\right]^{r_{m}}\right) \\
& +\left(\prod_{i=0}^{m-1}\left[w_{1}^{(i)}\right]^{r_{i}}-\prod_{i=0}^{m-1}\left[w_{2}^{(i)}\right]^{r_{i}}\right)\left[w_{2}^{(m)}\right]^{r_{m}} \\
= & \left(w_{1}^{(m)}-w_{2}^{(m)}\right) \prod_{i=0}^{m-1}\left[w_{1}^{(i)}\right]^{r_{i}} \sum_{i=0}^{r_{m}-1}\left[w_{1}^{(m)}\right]^{r_{m}-1-i}\left[w_{2}^{(m)}\right]^{i}
\end{aligned}
$$




$$
\begin{aligned}
& +\left(\prod_{i=0}^{m-1}\left[w_{1}^{(i)}\right]^{r_{i}}-\prod_{i=0}^{m-1}\left[w_{2}^{(i)}\right]^{r_{i}}\right)\left[w_{2}^{(m)}\right]^{r_{m}} \\
= & \left(w_{1}^{(m)}-w_{2}^{(m)}\right) M_{r, m-1}\left[w_{1}\right] \sum_{i=0}^{r_{m}-1}\left[w_{1}^{(m)}\right]^{r_{m}-1-i}\left[w_{2}^{(m)}\right]^{i} \\
& +\left(M_{r, m-1}\left[w_{1}\right]-M_{r, m-1}\left[w_{2}\right]\right)\left[w_{2}^{(m)}\right]^{r_{m}},
\end{aligned}
$$

by the induction hypothesis, we know that the case $m$ of Lemma 3 holds. Therefore Lemma 3 holds.

The proof of Lemma 3 is complete.

Lemma 4 [24] Let $f(z)$ be an elliptic function of order $m\left(m=q_{1}+\cdots+q_{l}\right)$ and with $l$ distinct poles $z_{1}, \ldots, z_{l}$ of multiplicity $q_{1}, \ldots, q_{l}$ per parallelogram of periods. Then

$$
\begin{aligned}
f(z)= & \sum_{i=1}^{l} \sum_{j=2}^{q_{i}} \frac{(-1)^{j} c_{-i j}}{(j-1) !} \frac{d^{j-2}}{d z^{j-2}}\left(\frac{1}{4}\left[\frac{\wp^{\prime}(z)+B_{i}}{\wp(z)-A_{i}}\right]^{2}-\wp(z)\right) \\
& +\sum_{i=1}^{l} \frac{c_{-i 1}}{2} \frac{\wp^{\prime}(z)+B_{i}}{\wp(z)-A_{i}}+c_{0},
\end{aligned}
$$

where $c_{-i j}$ is obtained by (1.2), $B_{i}^{2}=4 A_{i}^{3}-g_{2} A_{i}-g_{3}$ and $\sum_{i=1}^{l} c_{-i 1}=0$.

If 0 is a pole, say $z_{l}=0$, then (2.2) can be expressed as

$$
\begin{aligned}
f(z)= & \sum_{i=1}^{l-1} \sum_{j=2}^{q_{i}} \frac{(-1)^{j} c_{-i j}}{(j-1) !} \frac{d^{j-2}}{d z^{j-2}}\left(\frac{1}{4}\left[\frac{\wp^{\prime}(z)+B_{i}}{\wp(z)-A_{i}}\right]^{2}-\wp(z)\right) \\
& +\sum_{i=1}^{l-1} \frac{c_{-i 1}}{2} \frac{\wp^{\prime}(z)+B_{i}}{\wp(z)-A_{i}}+\sum_{j=2}^{q_{l}} \frac{(-1)^{j} c_{-l j}}{(j-1) !} \frac{d^{j-2}}{d z^{j-}} \wp(z)+c_{0} .
\end{aligned}
$$

In order to give the representations of elliptic solutions, we need some notations and results concerning elliptic function [24].

Let $\omega_{1}, \omega_{2}$ be two given complex numbers such that $\operatorname{Im} \frac{\omega_{1}}{\omega_{2}}>0, L=L\left[2 \omega_{1}, 2 \omega_{2}\right]$ be discrete subset $L\left[2 \omega_{1}, 2 \omega_{2}\right]=\left\{\omega \mid \omega=2 n \omega_{1}+2 m \omega_{2}, n, m \in \mathbb{Z}\right\}$, which is isomorphic to $\mathbb{Z} \times \mathbb{Z}$. The discriminant $\Delta=\Delta\left(c_{1}, c_{2}\right):=c_{1}^{3}-27 c_{2}^{2}$ and

$$
s_{n}=s_{n}(L):=\sum_{\omega \in L \backslash\{0\}} \frac{1}{\omega^{n}} .
$$

The Weierstrass elliptic function $\wp(z):=\wp\left(z, g_{2}, g_{3}\right)$ is a meromorphic function with double periods $2 \omega_{1}, 2 \omega_{2}$ and satisfies the equation

$$
\left(\wp^{\prime}(z)\right)^{2}=4 \wp(z)^{3}-g_{2} \wp(z)-g_{3}
$$

where $g_{2}=60 s_{4}, g_{3}=140 s_{6}$, and $\Delta\left(g_{2}, g_{3}\right) \neq 0$.

If we change (2.3a) to the form

$$
\left(\wp^{\prime}(z)\right)^{2}=4\left(\wp(z)-e_{1}\right)\left(\wp(z)-e_{2}\right)\left(\wp(z)-e_{2}\right),
$$

we have $e_{1}=\wp\left(\omega_{1}\right), e_{2}=\wp\left(\omega_{2}\right), e_{3}=\wp\left(\omega_{1}+\omega_{2}\right)$. 
Inversely, given two complex numbers $g_{2}$ and $g_{3}$ such that $\Delta\left(g_{2}, g_{3}\right) \neq 0$, there exist double periods $2 \omega_{1}, 2 \omega_{2}$ Weierstrass elliptic function $\wp(z)$ such that above relations hold.

It is easy to see that the set of poles of the Weierstrass elliptic function $\wp(z)$ is $L, \wp(z)$ has four distinct complete multiple values $e_{1}, e_{2}, e_{3}$ and infinite, and thus any other value of it must be simple.

Lemma $5[24,25]$ The Weierstrass elliptic functions $\wp(z):=\wp\left(z, g_{2}, g_{3}\right)$ have two successive degeneracies and addition formula as follows.

(I) Degeneracy to simply periodic functions (i.e., rational functions of one exponential $\left.e^{\alpha z}\right)$ according to

$$
\wp\left(z, 3 d^{2},-d^{3}\right)=2 d-\frac{3 d}{2} \operatorname{coth}^{2} \sqrt{\frac{3 d}{2}} z,
$$

if one root $e_{j}$ is double $\left(\Delta\left(g_{2}, g_{3}\right)=0\right)$.

(II) Degeneracy to rational functions of $z$ according to

$$
\wp(z, 0,0)=\frac{1}{z^{2}}
$$

if one root $e_{j}$ is triple $\left(g_{2}=g_{3}=0\right)$.

(III) Addition formula

$$
\wp\left(z-z_{0}\right)=-\wp(z)-\wp\left(z_{0}\right)+\frac{1}{4}\left[\frac{\wp^{\prime}(z)+\wp^{\prime}\left(z_{0}\right)}{\wp(z)-\wp\left(z_{0}\right)}\right]^{2} .
$$

By above lemmas, we can give a new method below, say the complex method, to find exact solutions of some PDEs.

Step 1. Substituting the transform $T: u(x, t) \rightarrow w(z),(x, t) \rightarrow z$ into a given PDE gives nonlinear ordinary differential equation (1.9).

Step 2. Substitute (1.2) into equation (1.9) to verify that the weak $\langle p, q\rangle$ condition and weight relations $p(r)+(q-1) \operatorname{deg}\left(M_{r}\right)<m+q$ hold

Step 3. By indeterminant relations (1.5), (1.6), and (1.7) we find the elliptic, rational and simply periodic solutions $w(z)$ of equation (1.9) with pole at $z=0$, respectively.

Step 4. By Theorem 1 we obtain all meromorphic solutions $w\left(z-z_{0}\right)$.

Step 5. Substitute the inverse transform $T^{-1}$ into these meromorphic solutions $w\left(z-z_{0}\right)$, then we get all exact solutions $u(x, t)$ of the original given PDE.

\section{Proof of the main theorem}

Proof of Theorem 1 Suppose that $w$ is a meromorphic solution of equation (1.9). We divide the proof into three cases.

Case 1. $w$ is entire.

We will first prove that $w$ is not transcendental. Otherwise, rewrite (1.9) as follows:

$$
w^{n-1} \cdot w=P[w]+a w^{(m)}
$$

Making use of Lemma 1, we get

$$
m(r, w)=S(r, w) .
$$


Noting that $N(r, w)=0$, we have

$$
T(r, w)=m(r, w)=S(r, w)=o(T(r, w))
$$

a contradiction. So $w$ is not a transcendental entire function.

Next, if $w$ is a polynomial of degree $k(k \geq 1)$, that is,

$$
w=a_{k} z^{k}+a_{k-1} z^{k-1}+\cdots+a_{0} \quad\left(a_{k} \neq 0\right) .
$$

Substituting $w$ into (1.9), we know that the coefficient $a_{k}^{n}$ of the highest degree term in $z$ is not zero. Hence $w$ is constant.

Case 2. $w$ is a meromorphic function with finite poles.

By using the logarithmic derivative lemma and properties of Nevanlinna's characteristic function, we have $T\left(r, \frac{w^{(i)}}{w}\right)=O(\log r)+S(r, w)$. Change $P[w]$ to the form of

$$
P[w]=\sum_{i=0}^{n-1} b_{i}\left(\frac{w^{\prime}}{w}, \ldots, \frac{w^{(m-1)}}{w}\right) w^{i}
$$

where each $b_{i}(i=1, \ldots, n-1)$ is its variables polynomial, then Lemma 2 gives

$$
T\left(r, P[w]+a w^{(m)}\right) \leq(n-1) T(r, w)+O(\log r)+S(r, w) .
$$

By Lemma 2 once more, we have $T\left(r, w^{n}\right)=n T(r, w)+O(1)$, and then $T(r, w)=S(r, w)+$ $O(\log r)$. Hence $w$ is rational.

Assume that $z_{1}, z_{2}, \ldots, z_{p+1}$ are of $p+1$ distinct poles of $w$ on the whole $\mathbb{C}$, then $w(z+$ $\left.z_{1}\right), \ldots, w\left(z+z_{p+1}\right)$ are solutions of equation (1.9) which have pole at $z=0$. By the weak $\langle p, q\rangle$ condition, we see that there exist at least two of them, say $w_{1}(z):=w\left(z+z_{1}\right)$ and $w_{2}(z):=w\left(z+z_{p+1}\right)$, such that $w_{1}(z)$ and $w_{2}(z)$ have the same Laurent principle parts. We will prove that $w_{1}(z) \equiv w_{2}(z)$. Otherwise, $z=0$ is a zero of $w_{1}(z)-w_{2}(z)$ with multiplicity $l \geq 0$. Substituting $w_{1}(z)$ and $w_{2}(z)$ into equation (1.9), we get

$$
a w_{i}^{(m)}+P\left[w_{i}\right]=w_{i}^{n}, \quad i=1,2,
$$

and

$$
a\left(w_{1}^{(m)}-w_{2}^{(m)}\right)+\left(P\left[w_{1}\right]-P\left[w_{2}\right]\right)=w_{1}^{n}-w_{2}^{n} .
$$

Since $z=0$ is a pole of $w_{i}$ with multiplicity $q$, by hypothesis $p(r)+(q-1) \operatorname{deg}\left(M_{r}\right)<m+q$ and $\operatorname{deg}(P)<n,(3.1)$ implies

$$
m=(n-1) q
$$

Thus, by (3.3), we can change (3.1) into the form

$$
a \prod_{j=1}^{m} \frac{z w_{i}^{(j)}}{w_{i}^{(j-1)}}+\frac{z^{m} P\left[w_{i}\right]}{w_{i}}=\left(z^{q} w_{i}\right)^{n-1}, \quad i=1,2
$$


Since $m+q-\left(\sum_{l=0}^{j}(q+l) r_{l}\right)>m+q-p(r)-(q-1) \operatorname{deg}\left(M_{r}\right)>0$, we get that for each $i=1,2$, as $z \rightarrow 0$,

$$
\begin{aligned}
z^{m+q} P\left[w_{i}\right] & =\sum_{r \in I} \sum_{j=0}^{m-1} a_{r, j} z^{m+q} M_{r, j}\left[w_{i}\right] \\
& =\sum_{r \in I} \sum_{j=0}^{m-1} a_{r, j} z^{m+q-\left(\sum_{l=0}^{j}(q+l) r_{l}\right)} \prod_{k=0}^{j}\left[z^{q} w_{i}^{(k)}\right]^{r_{k}} \rightarrow 0 .
\end{aligned}
$$

Hence, setting $z \rightarrow 0$ in (3.4) and combining $\operatorname{deg}(P)<n$, we have

$$
c_{q}^{n-1}=(-1)^{m} a q(q+1) \cdots(q+m-1) .
$$

By Lemma 3, (3.2) can be changed into the form

$$
\begin{aligned}
a\left(w_{1}^{(m)}-w_{2}^{(m)}\right)-\left(w_{1}^{n}-w_{2}^{n}\right) & =P\left[w_{1}\right]-P\left[w_{2}\right] \\
& =\sum_{r \in I} \sum_{j=0}^{m-1} a_{r, j}\left(M_{r, j}\left[w_{1}\right]-M_{r, j}\left[w_{2}\right]\right) \\
& =\sum_{r \in I} \sum_{j=0}^{m-1} a_{r, j}\left(\sum_{i=0}^{j}\left(w_{1}^{(i)}-w_{2}^{(i)}\right) P_{i, j}\left[w_{1}, w_{2}\right]\right),
\end{aligned}
$$

where each $P_{i, j}\left[w_{1}, w_{2}\right](i=0, \ldots, j, j=0, \ldots, m-1)$ is a homogenous differential polynomial in $w_{1}$ and $w_{2}$ such that the total weight

$$
W\left(P_{i, j}\right)=p(r)-i-1<W(P)-i-1 \leq m-i-2
$$

and degree

$$
\operatorname{deg}\left(P_{i, j}\right)=\operatorname{deg}\left(M_{r, j}\right)-1<\operatorname{deg}(P)-1 \leq n-2,
$$

respectively.

And then

$$
\begin{aligned}
a z^{m} & \prod_{i=1}^{m} \frac{\left(w_{1}-w_{2}\right)^{(i)}}{\left(w_{1}-w_{2}\right)^{i-1}}-\left(\sum_{i=0}^{n-1}\left(z^{q} w_{1}\right)^{i}\left(z^{q} w_{2}\right)^{n-1-i}\right) \\
= & \sum_{r \in I} \sum_{j=0}^{m-1} a_{r, j} z^{m+q-\left(\sum_{l=0}^{j}(q+l) r_{l}\right)} \\
& \times\left(\sum_{i=0}^{j} z^{i} \frac{\left(w_{1}-w_{2}\right)^{(i)}}{w_{1}-w_{2}} z^{-q-i+\sum_{l=0}^{j}(q+l) r_{l}} P_{i, j}\left[w_{1}, w_{2}\right]\right) .
\end{aligned}
$$

Noting that $P_{i, j}\left[w_{1}, w_{2}\right]=\prod_{k=0}^{i-1}\left[w_{1}^{(k)}\right]^{r_{k}} \prod_{k=i+1}^{j}\left[w_{2}^{(k)}\right]^{r_{k}}\left(\sum_{k=0}^{r_{i}-1}\left[w_{1}^{(i)}\right]^{k}\left[w_{2}^{(i)}\right]^{r_{i}-1-k}\right)(i=0, \ldots, j)$ and $m+q-\left(\sum_{l=0}^{j}(q+l) r_{l}\right)>m+q-p(r)-(q-1) \operatorname{deg}\left(M_{r}\right)>0$, by the similarly argument and taking $z \rightarrow 0$, (3.7) implies

$$
n c_{q}^{n-1}=\operatorname{al}(l-1) \cdots(l-m+1) .
$$


Since $m$ is an odd integer, from both (3.5) and (3.8), we infer $c_{q}=0$. This is impossible. Thus $w(z) \equiv w\left(z-z_{1}+z_{p+1}\right)$, hence $w$ is periodic. But a periodic rational function must be constant, a contradiction.

Therefore $w$ has at most $l$ distinct poles on $\mathbb{C}$, say $l \leq p$ distinct poles $z_{1}, \ldots, z_{l}$. Furthermore, $w$ has the form of

$$
w(z)=\sum_{i=1}^{l} \sum_{j=1}^{q} \frac{c_{i j}}{\left(z-z_{i}\right)^{j}}+P(z),
$$

where $P(z)$ is a polynomial. Substituting (3.9) into equation (1.9), computing the indeterminant coefficients from the highest term in turn in $z$ for $P(z)$ by the hypothesis, we infer that $P(z)$ is constant. Thus (I) occurs.

Case 3. $w$ has infinite poles.

From former conclusion, we know that there exist $l \leq p$ distinct poles $z_{1}, \ldots, z_{l}$ such that the set of all poles of $w$ can be expressed as $z_{1}+\Gamma, \ldots, z_{l}+\Gamma$, where $\Gamma$ is a nontrivial discrete subset on $(\mathbb{C},+)$. Thus $\Gamma$ is isomorphic $\mathbb{Z}$, or isomorphic $\mathbb{Z} \times \mathbb{Z}$. We consider them, respectively.

If $\Gamma$ is isomorphic $\mathbb{Z}$, then $\mathbb{C} / \Gamma=\mathbb{C}^{*}=\mathbb{C} \backslash 0$, and $w$ is a single periodic function. Then $w=R(\exp (\alpha z))$, where $R$ is a meromorphic function with $l(\leq p)$ distinct poles $\xi_{1}, \ldots, \xi_{l}$ of multiplicity $q$. We will prove that $R$ is a rational function.

Set $\xi=\exp (\alpha z)$ and substitute $w=R(\xi)$ into equation (1.9), we have

$$
a \alpha^{m}\left[\xi R^{\prime}+\cdots+\xi^{m} R^{(m)}\right]+P[R]=R^{n} .
$$

It is the same argument as Case 1, if $R$ is transcendental, Lemma 1 implies $m(r, R)=$ $S(r, R)$. Noting that $R$ has and only has $l \leq p$ distinct poles, we have $T(r, R)=S(r, R)$, a contradiction. Therefore, $R$ is a rational function.

By a similar argument of the later part of Case 2 for $R$ and equation (3.10), we see that (II) holds.

If $\Gamma$ is isomorphic $\mathbb{Z} \times \mathbb{Z}$, then $w$ is an elliptic function and has $l(\leq p)$ distinct poles $\xi_{1}, \ldots, \xi_{l}$ of multiplicity $q$ per parallelogram of periods.

(1.7) can be obtained from (2.3) of Lemma 4. By Lemma 4, we can obtain another representation of $w$.

(1) If $w$ is even. By Lemma 4, we know that $w$ can be expressed as $w=Q(\wp(z))$, where $Q(\xi)$ is a rational function. Noting that the poles of $w$ are of multiplicity $q$ and the poles of $\wp(z)$ are of multiplicity 2, we infer that $Q(\xi)$ has the form of

$$
Q(\xi)=\sum_{i=1}^{l} \sum_{j=1}^{q} \frac{c_{i j}}{\left(\xi-\xi_{i}\right)^{j}}+\sum_{i=0}^{q_{0}} c_{i} \xi^{i}
$$

where $q_{0} \leq \frac{q}{2}$ and the pole of $\wp(z)$ is the pole of $w$, or then $q_{0}=0$ if the pole of $\wp(z)$ is not the pole of $w$.

(2) If $w$ is odd, note that $\wp^{\prime}(z)$ is odd, then $\frac{w}{\wp^{\prime}(z)}$ is even. By conclusion (1), we deduce that $\frac{w}{\wp^{\prime}(z)}$ is a rational $Q(\xi)$ of $\xi:=\wp(z)$.

(3) If $w$ is non-odd and non-even, then rewrite $w$ to the form of $w(z)=\frac{w(z)+w(-z)}{2}+$ $\frac{w(z)-w(-z)}{2}$. It is easy to know that $\frac{w(z)+w(-z)}{2}$ is even, and $\frac{w(z)-w(-z)}{2}$ is odd. Therefore both 
conclusions (1) and (2) give

$$
w(z)=Q_{1}(\wp(z))+\wp(z)^{\prime} Q_{2}(\wp(z))
$$

where $Q_{1}(\xi)$ and $Q_{2}(\xi)$ are rational functions with the form of (3.11). Conclusion (3) holds.

Thus (III) occurs.

The proof of Theorem 1 is complete.

\section{All meromorphic solutions of the Kuramoto-Sivashinsky equation}

In this section, we give an example to show our complex method and the application of Theorem 1.

Substituting (1.2) into equation (1.3), we have

$$
w(z)=\frac{120 v}{z^{3}}-\frac{15 b}{z^{2}}+\left(\frac{60 \mu}{19}-\frac{15 b^{2}}{76 v}\right) \frac{1}{z}+\cdots
$$

Thus $q=3, p=1, c_{-3}=120 v, c_{-2}=-15 b, c_{-1}=\left(\frac{60 \mu}{19}-\frac{15 b^{2}}{76 v}\right)$.

Hence, equation (1.3) satisfies the weak $\langle 1,3\rangle$ condition. Obviously, equation (1.3) satisfies other conditions. So, by Theorem 1, we know that all meromorphic solutions of equation (1.3) belong to $W$. Now we will give the forms of all meromorphic solutions of equation (1.3).

By (2.3) of Lemma 4, we infer that the indeterminant of elliptic solutions with pole $z=0$ is

$$
w_{d 0}(z)=-\frac{c_{-3}}{2} \wp^{\prime}(z)+c_{-2} \wp(z)+c_{0}
$$

where $\wp(z)$ is the Weierstrass elliptic function, $c_{-3}=120 v, c_{-2}=-15 b, c_{0}$ indeterminant.

Substituting (4.1) into equation (1.3), we have $b^{2}=16 v \mu$.

Therefore, all elliptic function solutions of equation (1.3) are

$$
w_{d}(z)=-60 \nu \wp^{\prime}\left(z-z_{0}\right)-15 b \wp\left(z-z_{0}\right) \text {, }
$$

where $b^{2}=16 v \mu$. Making use of addition formula (2.6) of Lemma 5, we can rewrite it into the form

$$
\begin{aligned}
w_{d}(z) & \\
= & 15 b\left(\wp(z)+\wp\left(z_{0}\right)-\frac{1}{4}\left[\frac{\wp^{\prime}(z)+\wp^{\prime}\left(z_{0}\right)}{\wp(z)-\wp\left(z_{0}\right)}\right]^{2}\right) \\
& +60 \nu\left(\wp^{\prime}(z)+\frac{1}{2} \frac{\left(\wp^{\prime}(z)+\wp^{\prime}\left(z_{0}\right)\right)\left[\wp^{\prime \prime}(z)\left(\wp(z)-\wp\left(z_{0}\right)\right)-\wp^{\prime}(z)\left(\wp^{\prime}(z)+\wp^{\prime}\left(z_{0}\right)\right)\right]}{\left(\wp(z)-\wp\left(z_{0}\right)\right)^{3}}\right),
\end{aligned}
$$

where $z_{0} \in \mathbb{C}, b^{2}=16 v \mu$. 
Making using of (2.5) of Lemma 5, all simply periodic function solutions of equation (1.3) with pole $z=0$ can be degenerated by

$$
\begin{aligned}
w_{s 0}(z)= & -60 v \wp^{\prime}\left(z, 3 d^{2},-d^{3}\right)-15 b \wp\left(z, 3 d^{2},-d^{3}\right) \\
= & 180 v d \sqrt{\frac{3 d}{2}}\left(\operatorname{coth}^{2} \sqrt{\frac{3 d}{2}} z-1\right) \operatorname{coth} \sqrt{\frac{3 d}{2}} z \\
& +\frac{45 b d}{2} \operatorname{coth}^{2} \sqrt{\frac{3 d}{2}} z-30 b d .
\end{aligned}
$$

Thus, we have all simply periodic function solutions

$$
\begin{aligned}
w_{s}(z)= & 180 v d \sqrt{\frac{3 d}{2}}\left(\operatorname{coth}^{2} \sqrt{\frac{3 d}{2}}\left(z-z_{0}\right)-1\right) \operatorname{coth} \sqrt{\frac{3 d}{2}}\left(z-z_{0}\right) \\
& +\frac{45 b d}{2} \operatorname{coth}^{2} \sqrt{\frac{3 d}{2}}\left(z-z_{0}\right)-30 b d
\end{aligned}
$$

where $z_{0} \in \mathbb{C}, d \neq 0$.

Making use of (2.6) of Lemma 5, all rational function solutions of equation (1.3) with pole $z=0$ can be degenerated by

$$
\begin{aligned}
w_{r 0}(z) & =-60 v \wp^{\prime}(z, 0,0)-15 b \wp(z, 0,0) \\
& =\frac{120 v}{z^{3}}-\frac{15 b}{z^{2}} .
\end{aligned}
$$

Putting (4.2) into (1.3), we deduce that $A=b=\mu=0$. Hence, we have all rational function solutions

$$
w_{r}(z)=\frac{120 v}{\left(z-z_{0}\right)^{3}},
$$

where $z_{0} \in \mathbb{C}, A=b=\mu=0$.

\section{Conclusions}

The complex method is very important in finding the exact solutions of nonlinear evolution equations, and AODEq. (1.9) is a class of most important auxiliary equations because many nonlinear evolution equations can be converted to this equation making use of the traveling wave reduction. In this article, we employ Nevanlinna's value distribution theory to investigate the existence of meromorphic solutions of some algebraic differential equations. We obtain the representations of all meromorphic solutions of certain algebraic differential equations with constant coefficients and dominant term. Many results are the corollaries of our result, and we will give the complex method to find all traveling wave exact solutions of corresponding partial differential equations. Our results show that the complex method provides a powerful mathematical tool for solving great many nonlinear partial differential equations in mathematical physics. 


\section{Authors' contributions}

WY carried out the main part of this manuscript. JQ and YL participated in discussion and corrected the main theorem. All authors read and approved the final manuscript.

\section{Author details}

${ }^{1}$ School of Mathematics and Information Science, Guangzhou University, Guangzhou, 510006, China. ${ }^{2}$ Key Laboratory of Mathematics and Interdisciplinary Sciences of Guangdong Higher Education Institutes, Guangzhou University, Guangzhou, 510006, China. ${ }^{3}$ School of Science, Beijing University of Posts and Telecommunications, Beijing, 100876 , China. ${ }^{4}$ Department of Mathematics and Physics, Shanghai Dianji University, Shanghai, 201306, China.

\section{Acknowledgements}

The first author would like to express his hearty thanks to Professor Fang Mingliang and Liao Liangwen for their helpful discussions and suggestions. This work was supported by the Visiting Scholar Program of Chern Institute of Mathematics at Nankai University when the authors worked as visiting scholars. The authors would like to express their hearty thanks to Chern Institute of Mathematics for very comfortable research environment. This work was supported by the NSF of China (11271090), Tianyuan Youth Fund of the NSF of China (11326083), Shanghai University Young Teacher Training Program (ZZSDJ12020), Innovation Program of Shanghai Municipal Education Commission (14YZ164), the NSF of Guangdong Province (S2012010010121), Project 13XKJC01 from the Leading Academic Discipline Project of Shanghai Dianji University.

\section{Received: 5 January 2014 Accepted: 16 March 2014 Published: 4 April 2014}

\section{References}

1. Ablowitz, MJ, Clarkson, PA: Solitons, Nonlinear Evolution Equations and Inverse Scattering. London Mathematical Society Lecture Note Series. Cambridge University Press, Cambridge (1991)

2. Matveev, VB, Salle, MA: Darboux Transformations and Solitons. Springer Series in Nonlinear Dynamics. Springer, Berlin (1991)

3. Hirota, R, Satsuma, J: Soliton solutions of a coupled KdV equation. Phys. Lett. A 85, 407-408 (1981)

4. Olver, PJ: Applications of Lie Groups to Differential Equations. Graduate Texts in Mathematics. Springer, New York (1993)

5. Li, JB, Liu, Z: Travelling wave solutions for a class of nonlinear dispersive equations. Chin. Ann. Math. 23, 397-418 (2002)

6. Tang, S, Huang, W: Bifurcations of travelling wave solutions for the generalized double sinh-Gordon equation. Appl. Math. Comput. 189, 1774-1781 (2007)

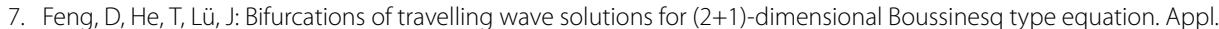
Math. Comput. 185, 402-414 (2007)

8. Tang, S, Xiao, Y, Wang, Z: Travelling wave solutions for a class of nonlinear fourth order variant of a generalized Camassa-Holm equation. Appl. Math. Comput. 210, 39-47 (2009)

9. Tang, S, Zheng, J, Huang, W: Travelling wave solutions for a class of generalized KdV equation. Appl. Math. Comput. 215, 2768-2774 (2009)

10. Malfliet, W, Hereman, W: The tanh method: I. Exact solutions of nonlinear evolution and wave equations. Phys. Scr. 54, 563-568 (1996)

11. Fan, E: Uniformly constructing a series of explicit exact solutions to nonlinear equations in mathematical physics. Chaos Solitons Fractals 16, 819-839 (2003)

12. Wang, ML: Solitary wave solutions for variant Boussinesq equations. Phys. Lett. A 199, 169-172 (1995)

13. Yang, L: Value-Distribution Theory and Its New Research. Science Press, Beijing (1982)

14. Eremenko, A: Meromorphic traveling wave solutions of the Kuramoto-Sivashinsky equation. Zh. Mat. Fiz. Anal. Geom. 2, 278-286 (2006)

15. Eremenko, A: Meromorphic solutions of equations of Briot-Bouquet type. Teor. Funkc. Funkc. Anal. Ih Prilozh. 38 48-56 (1982) English translation: Amer. Math. Soc. Transl. 133(2), 15-23 (1986)

16. Eremenko, A, Liao, LW, Ng, TW: Meromorphic solutions of higher order Briot-Bouquet differential equations. Math Proc. Camb. Philos. Soc. 146, 197-206 (2009)

17. Yuan, WJ, Shang, YD, Huang, Y, Wang, H: The representation of meromorphic solutions of certain ordinary differential equations and its applications. Sci. Sin. Math. 43(6), 563-575 (2013). doi:10.1360/012012-159

18. Conte, R, Ng, TW: Meromorphic solutions of a third order nonlinear differential equation. J. Math. Phys. 51, 033518 (2010)

19. Kuramoto, Y, Tsuzuki, T: Persistent propagation of concentration waves in dissipative media far from thermal equilibrium. Prog. Theor. Phys. 55, 356-369 (1976)

20. Kudryashov, NA: Exact soliton solutions of the generalized evolution equation of wave dynamics. Prikl. Mat. Meh. 52, 465-470 (1988) English transl.: J. Appl. Math. Mech. 52, 361-365 (1988)

21. Kudryashov, NA: Exact solutions of the generalized Kuramoto-Sivashinsky equation. Phys. Lett. A 147, $287-291$ (1990)

22. Laine, I: Nevanlinna Theory and Complex Differential Equations. de Gruyter, Berlin (1993)

23. He, YZ, Xiao, XZ: Algebroid Functions and Ordinary Differential Equations. Science Press, Beijing (1988)

24. Lang, S: Elliptic Functions, 2nd edn. Springer, New York (1987)

25. Conte, R, Musette, M: Elliptic general analytic solutions. Stud. Appl. Math. 123(1), 63-81 (2009)

doi:10.1186/1687-1847-2014-105

Cite this article as: Yuan et al.: All meromorphic solutions of some algebraic differential equations and their applications. Advances in Difference Equations 2014 2014:105. 